\title{
Applicability of artificial intelligence models
}

\author{
Michele Tomaiuolo ${ }^{1}$
}

Published online: 10 August 2020

(C) Springer-Verlag London Ltd., part of Springer Nature 2020

In the last decade, great theoretical advancements have been obtained in Artificial Intelligence, especially in the field of machine learning but also in more traditional branches. The progresses have been demonstrated by important results in many paradigmatic case studies. However, these models and results need to be confirmed in more applications and their practical usefulness has be tested in various domains, to find their specific strenghts and limitations. In this sense, the current landscape of research works conducted in Artificial Intelligence is very lively and fertile, with new achievements obtained continuously.

This special issue is based upon some of the best researches presented at the Artificial Intelligence International Conference (A2IC-2018), held in Barcelona in 2018. As such, its aim is to join both academy and industry, considering research works that bring together theory and applications, but also considering ethical and philosofical knots, some of which are born together with the very notion of Artificial Intelligence. In this sense, it promotes exacly the type of experimentation which is indispensible for understanding the concrete applicability of new models to different realms.

Among the most promising algorithms and frameworks, many are based on Artificial Neural Networks (ANNs) and Deep Neural Networks (DNNs). In fact they are being applied to very different domains, such as computer vision, audio and streaming data, Natural Language Processing, etc. However, the best results in each domain are obtained by different kinds of models, such as Convolutional Neural Networks (CNNs), Recurrent Neural Networks (RNNs), Long Short-Term Memory (LSTM), Attention Networks and Transformers [8]. At the same time, also other consolidated AI concepts, such as ontologies, lexicons,

Michele Tomaiuolo

michele.tomaiuolo@unipr.it

1 Department of Engineering and Architecture, University of Parma, Parma, Italy consensus and trust, are being used in innovative ways to create practical systems. In this special issue, [6] provides some interenting examples. More in general, for many complex tasks, software systems engineering princicples can be applied effectively, for realizing structured architectures [2,5] and hierarchical systems [1, 4]. As shown in [3], also in this special issue, often higher level features have to be first encoded by lower level components, starting from raw data, before they can be used for obtaining the final results.

In particular, the first included article [6] tackles the important task of automatic recommendations, through a structured process. The work is interesting since it avoids machine learning altogether. Instead, after joining ratings, written reviews, and the result of lexicon-based sentiment analysis [7], it builds on Hesitant Fuzzy Linguistic Term Sets (HFLTSs) [9]. This approach, which reflects the hesitancy inherent in human reasoning, is used for group decision making. In fact, the proposed system aims at introducing a measure of consensus about the items to suggest, based on the agreement level of a large number of reviewers. This way, users are helped to differentiate among the suggested items.

The second article [3] deals with the highly critical task of cancer type prediction. The analysis is based on the notion of Copy Number Variations (CNVs), i.e., the presence of gene or genomic regions in different number of copies [10]. For analyzing such high dimensional data effectively, a hierarchical learning process is realized. An interesting neural ensemble method is applied. At the lower level, both deep neural networks and autoencoders derive more complex high-level features from input data, capturing contextual information. The effect is that the system at this layer learns non-linear mappings. Thus, at the higher level, features can be encoded in a lower-dimentional space, with respect to the original input data space, allowing more effective diagnosis. 


\section{References}

1. Angiani G, Cagnoni S, Chuzhikova N, Fornacciari P, Mordonini M, Tomaiuolo M (2016) Flat and hierarchical classifiers for detecting emotion in tweets. Lect Notes Artif Intell 10037:51-64. https://doi.org/10.1007/978-3-319-49130-1_5

2. Fornacciari P, Mordonini M, Poggi A, Sani L, Tomaiuolo M (2018) A holistic system for troll detection on twitter. Comput Hum Behav 89:258-268. https://doi.org/10.1016/j.chb.2018.08. 008

3. Karim MR, Rahman A, Jares JB, Decker S, Beyan O (2019) A snapshot neural ensemble method for cancer-type prediction based on copy number variations. Neural Comput Appl. https:// doi.org/10.1007/s00521-019-04616-9

4. Lombardo G, Fornacciari P, Mordonini M, Sani L, Tomaiuolo M (2019) A combined approach for the analysis of support groups on facebook - the case of patients of hidradenitis suppurativa. Multimedia Tools Appl 78(3):3321-3339. https://doi.org/10. 1007/s11042-018-6512-5

5. Lombardo G, Fornacciari P, Mordonini M, Tomaiuolo M, Poggi A (2019) A multi-agent architecture for data analysis. Future Internet. https://doi.org/10.3390/fi11020049
6. Nguyen J, Montserrat-Adell J, Agell N, Sánchez M, Ruiz FJ (2020) Fusing hotel ratings and reviews with hesitant terms and consensus measures. Neural Comput Appl. https://doi.org/10. 1007/s00521-020-04778-x

7. Nielsen FA (2011) A new anew: evaluation of a word list for sentiment analysis in microblogs. In: 1st workshop on making sense of microposts, pp 93-98

8. Pouyanfar S, Sadiq S, Yan Y, Tian H, Tao Y, Reyes MP, Shyu ML, Chen SC, Iyengar S (2018) A survey on deep learning: algorithms, techniques, and applications. ACM Comput Surv (CSUR) 51(5):1-36. https://doi.org/10.1145/3234150

9. Rodriguez RM, Martinez L, Herrera F (2011) Hesitant fuzzy linguistic term sets for decision making. IEEE Trans Fuzzy Syst 20(1):109-119

10. Tomczak K, Czerwińska P, Wiznerowicz M (2015) The cancer genome atlas (tcga): an immeasurable source of knowledge. Contemp Oncol 19(1A):A68

Publisher's Note Springer Nature remains neutral with regard to jurisdictional claims in published maps and institutional affiliations. 